\title{
Cell Damages and Reperfusion Effects on Postischemic Hippocampus of Gerbils
}

\author{
S.M. Yu, ${ }^{*}$ S.W. Wu, ${ }^{* *}$ and K.H. Lin***
}

* Department of Nursing, College of Nursing, Central Taiwan University of Science and Technology, Taichung, 406 Taiwan.

** Institute of Anatomy and Cell Biology, National Yang-Ming University, Taipei, 112 Taiwan.

*** Graduate Institute of Physical Therapy, School of Physical Therapy, National Taiwan University, Taipei, 100 Taiwan.

Ischemia may result from impair of cerebral blood flow and lead to hypoxia and hypoglycemia, which cause pathological damage of brain. The hippocampus is the most vulnerable area to ischemia injury, and often used as an experimental model. In spite of the hippocampal CA1 presenting the delayed neuronal death after ischemia, the CA3 is more resistant. But the mechanisms are not fully understood, especially the morphological changes. The Mongolian gerbil, Meriones unguiculatus, were anesthetized with $\alpha$-chloralose $(350 \mathrm{mg} / \mathrm{kg}$ i.p. ) . The animals were subjected to the unilateral or bilateral common carotid arteries occlusion by a microvascular/miniature aneurismal clip. After 90 minutes of ischemia, the aneurismal clip on the common carotid arteries were removed to allow reperfusion. At 0, 30 minutes, 1, 2, 4 and 6 hours after reperfusion, animals were perfused with $6 \%$ paraformaldehyde-lysine- periodate in $0.1 \mathrm{M}$ phosphate buffer. After immersed the brain in the same fixative, the hippocampus was removed and then embedded in the Epon. For light microscopy, semithin sections $(1 \mu \mathrm{m})$ were cut with an ultramicrotome. The section was stained with toluidine blue. For EM, ultrathin sections $(90 \mathrm{~nm})$ were cut, stained with lead citrate, and examined under the electron microscope.

At post-reperfusion 30 minutes (Fig. 1), the clumped chromatin became more evident in the nucleus. Electron-lucent membrane-bound vacuoles frequently contained membranous structures. At postreperfusion 1 hour (Fig. 2), numerous small vesicles were accumulated in clusters associated with the endoplasmic reticulum. At post-reperfusion 2 hours (Fig. 3), a lot of electron-lucent large vacuoles accumulated and apposed to the peripheral cytoplasm. The perineuronal vacuoles were membrane-bound and coalesced to form a dumbbell-shaped vacuole. At post-reperfusion 4 hours (Fig. 4), clumped chromatins were apposed to the nucleolemma or randomly distributed in the nucleus. The cytoplasmic organelles, nuclear membrane and matrix were disintegrated.

FIG 1. TEM micrograph of the CA3 at post-reperfusion 30 minutes. Note conspicuous nucleolus (n), condensed nucleolus (double arrows) and clumped chromatin (arrow).

FIG 2. TEM micrograph of the CA3 at post-reperfusion 1 hour. Note clumped chromatins (arrow) and small vesicles (double arrowheads).

FIG 3. TEM micrograph of the CA3 at post-reperfusion 2 hours. Note electron-lucent large vacuoles (V) and dumbbell-shaped vacuole (arrowhead).

FIG 4. TEM micrograph of the CA3 at post-reperfusion 4 hours. Note clumped chromatins (arrow), mitochondria $(\mathrm{m})$ and perineuronal vacuoles $(\mathrm{V})$. Scale bar $=2 \mu \mathrm{m}$. 

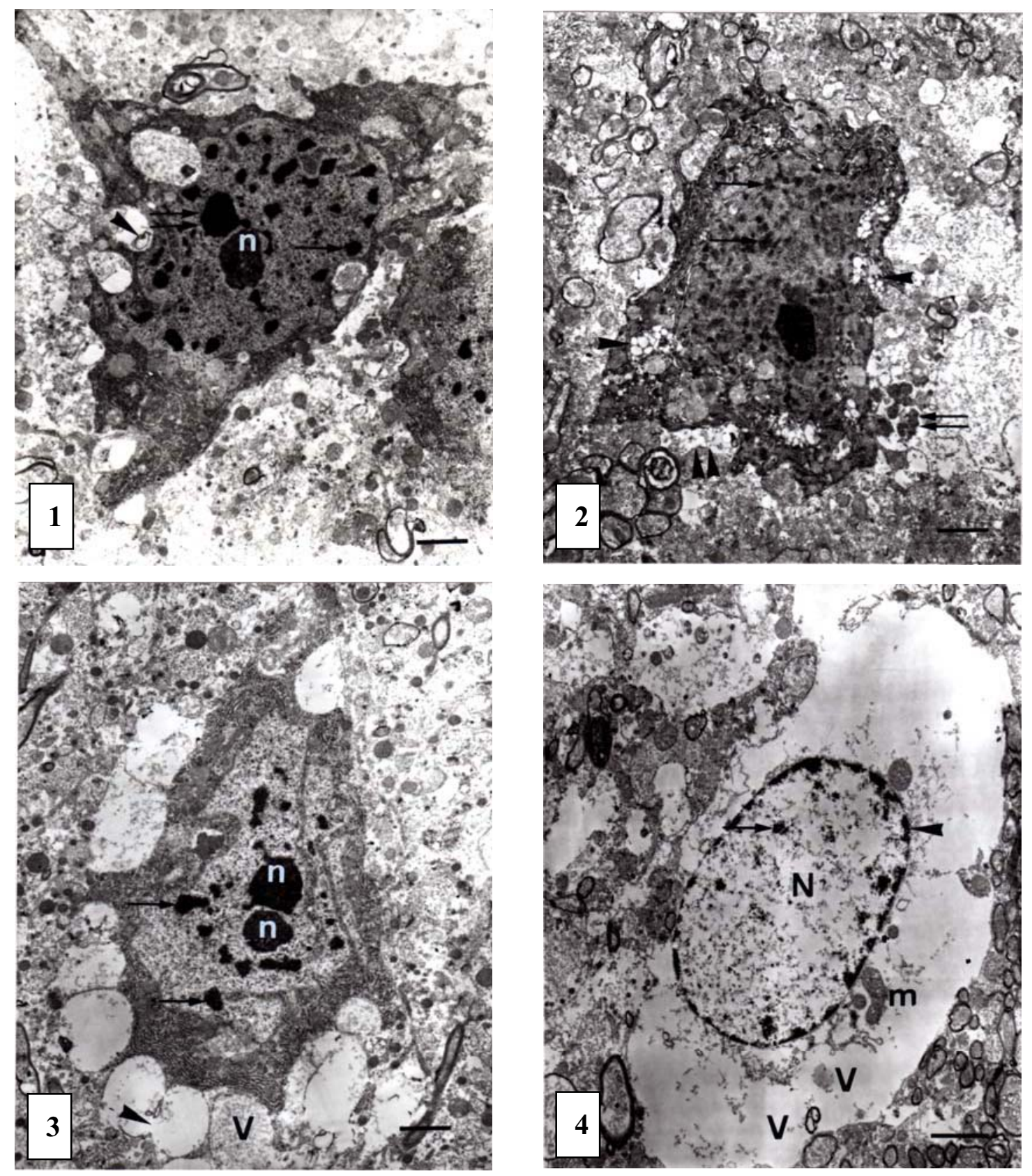